\section{Pattern perception: A predictive variable based on discriminability and selective attention}

\author{
CYNTHIA M. MAVRIDES, Laurentian \\ University, Sudbury, Ontario, Canada
}

Three classes of pattern measures were examined as predictors of latency in a five-choice discrimination task with $L$-shaped linear patterns. Those reflecting solely characteristics of the pattern were proved inapplicable (i.e., were not significantly related to latency) as were the measures reflecting only characteristics of the domain. Measures reflecting the relationship of each pattern to its domain proved applicable, and the best prediction of latency was obtained when a measure was generated based on the selective shift of attention to the maximum discriminating dimension in any single problem.

Several models and analytic processes are presently extant that provide different theoretical approaches to the existence of multiple dimensions in a visual pattern of stimulation. Specifically, it has been suggested (Mavrides \& Brown, 1969) that a pattern may be viewed as an aggregate of objects or events that are multidimensional, where the stimuli for perception are the quantities of the particular features to which the $S$ is sensitive or, in terms of Brunswick's theory, a pattern may be considered as being embedded in multidimensional space where the cue dimensions are defined by the ordering of patterns with respect to their perceptually relevant features (Beach, 1964a,b). Multidimensional analytic models incorporating responses to patterns have been suggested by Torgerson (1958) and Kruskal (1964a,b), among others, and have given rise to an accumulating body of data that support the contention that Ss encode forms in terms of specific features (Aiken \& Brown, 1969; Behrman \& Brown, 1968; Brown \& Andrews, 1968; Stenson, 1968).

It has been argued (Brown \& Owen, 1967; Evans, 1967) and later demonstrated (Mavrides \& Brown, 1969; Edmonds \& Evans, 1966) that the effective stimuli for perception depend on certain characteristics of the pattern domain (sample or population) in which the pattern is presented. The variance of dimensions in the domain was found to be a major contributor to pattern discriminability and stimulus memory.

In a review and analysis of various types of selective attention (Egeth, 1967), selection of one pattern dimension from the several presented to $S$ at one point in time has been documented and discussed at length. In particular, it was made obvious by a series of experiments (Fitts \& Biederman, 1967; Imai \& Garner, 1965; Morin, Forrin, \& Archer, 1961) that Ss were capable of effective gating of irrelevant information (i.e., were able to base responses in a classification task almost exclusively on variation in relevant dimensions). The Ss' dominant preferences and variation in the nonpreferred dimensions were both utilized in a free-classification task, while only discriminability of the chosen dimension was relevant in a constrained task (Imai \& Garner, 1965). For the constrained classification in particular, all irrelevant information and variation was filtered out and did not affect the speed with which Ss accomplished their task.

The experiment reported below was designed to demonstrate the presence of selective attention (and gating of irrelevant information) in responses to two-dimensional patterns. It is suggested that differences in attention will, when considered as a variable in response prediction, produce a more efficient model than those that are based solely on the two dimensions or on the variation in the pattern domain.

\section{PATTERNS}

The patterns were composed of two black lines on a white background. The lines (one vertical and the other horizontal) met at a 90-deg angle to form a shape resembling the letter $\mathrm{L}$. Either line could vary in length from $1 / 4$ to $6 / 4$ in. Three sets of patterns were generated, each including five patterns. Each set was generated by random selection of heights of $1 / 4$ to $6 / 4 \mathrm{in}$., and three sets were included in order to introduce differences in the $\mathrm{VarV}$ and $\mathrm{VarH}$ measures described below. An additional three sets were obtained by exchanging the vertical and horizontal components of the original patterns within each set. The task involved identifying each pattern in turn from among all patterns in the set. The pattern to be identified was placed below a row containing all patterns of the set in random order, creating a five-choice discrimination problem. Seven measures were then calculated for each pattern.

\section{Vertical Extent (V)}

$\mathrm{V}$ was calculated as the vertical height (expressed in $1 / 4$ in.).
Horizontal Extent $(\mathrm{H})$

$\mathrm{H}$ was calculated as the horizontal length.

Vertical Deviation (dV)

This and the following measure were included to evaluate the importance of differences of the pattern to be identified from other patterns in the set; $d V$ was calculated as equal to the sum of the differences between $\mathrm{V}$ of the pattern to be identified and all other patterns in the set.

Horizontal Deviation (dH)

$\mathrm{dH}$ was calculated similarly to $\mathrm{dV}$. Maximum Deviation (dmax)

dmax was designed to tap selective attention processes. It was hypothesized that Ss faced with any one discrimination problem would utilize the dimension providing maximum discriminability. In other words, the larger of $\mathrm{dV}$ and $\mathrm{dH}$ would be utilized while the smaller would be essentially ignored. The dmax value for any pattern, therefore, was the maximum of $\mathrm{dV}$ and $\mathrm{dH}$.

\section{Total Deviation (dT)}

If $S$ were neither using a constant dimension nor depending on selection of dimensions, it is possible that the ease of identification would depend on the total discriminability of any pattern from its set, in other words, on the sum of $\mathrm{dV}$ and $\mathrm{dH}$. Vertical Variation (VarV)

VarV was independent of the individual patterns, and was calculated as the second moment of $\mathrm{V}$ for any pattern set. $\mathrm{VarV}$ was therefore constant for all patterns in any single set.

\section{Horizontal Variation (VarH)}

VarH was similarly calculated for $\mathrm{H}$ values in any set.

These two latter measures were estimates applying only to the pattern domain, while $\mathrm{V}$ and $\mathrm{H}$ applied uniquely to the pattern, and $\mathrm{dV}, \mathrm{dH}, \mathrm{dmax}$, and $\mathrm{dT}$ were estimates applying to the pattern in relationship to the domain.

With relevance to the particular patterns used, a pilot study involving $10 \mathrm{Ss}$ did not significantly document the presence of the vertical-horizontal illusion. This is in partial agreement with Dember (1960), who mentions that the illusion is reduced when the lines do not bisect each other.

\section{METHOD \\ Subjects}

The Ss were nine volunteer college students.

Procedure

The Ss entered a darkened experimental cubicle. They were told by $\mathrm{E}$ that their task involved the identification of one L-shaped form from a set of five such forms that would appear above it. The problems were presented in slide form and were projected on a screen approximately $4 \mathrm{ft}$ away from the $\mathrm{S}$ at eye level. The 
dimensions mentioned in the previous section $(1 / 4 \mathrm{in}$.) were relevant. The presentation of a slide triggered a clock by means of a light-sensitive relay. Ss placed their fingers on a telegraph key before each slide was presented and raised it when they had made the identification. At this point, the clock was stopped and $\mathrm{E}$ terminated the presentation of the slide. Ss indicated the position of the correct pattern on a response sheet lying before them, and problems wrongly solved were presented again later in the series. Ss were presented with all 30 problems (six sets with five patterns per set) in random order after having received one practice trial with an extra-task problem. After a 3-min interval, Ss performed the total task a second time, and generally spent $10-12 \mathrm{~min}$ completing both administrations.

\section{RESULTS}

Table 1 includes the means and standard deviations of all seven measures. When each $S$ was represented by his mean latency (over problems), the mean latency over Ss for the first administration was $1.55 \mathrm{sec}$, while that for the second was $1.52 \mathrm{sec}$. Ss had consistent latencies across administrations (the correlation was .57), and $a t$ test performed on the mean latency for the first and the second administrations did not reject the hypothesis that the two means were equal $(\mathrm{p}>.05)$. This suggests either that no learning was involved in the task or that any learning that was present occurred in the early trials of the first administration. Because of these findings, each $S$ was represented by the average of his latencies on any one problem, and all Ss were represented by the mean of the nine individual means for any one problem. Each pattern, therefore, gave rise to one problem that could be quantified in terms of seven measures on the pattern and the domain, and one measure of latency averaged over Ss, the last being naturally the dependent measure.

Another indication of consistency was calculated when the mean latencies over all Ss were compared for the 15 original patterns and the 15 inverted patterns (where $\mathrm{V}$ and $\mathrm{H}$ were interchanged). Responses correlated significantly (.43)

Table 1

Means and SDs for the Seven Measures of Pattern and the Domain and for Discrimination Latency (Averaged Over Ss)

\begin{tabular}{lcl}
\hline Variables & Mean & \multicolumn{1}{c}{ SD } \\
\hline $\mathrm{V}$ & 3.50 & $1.73(1 / 4 \mathrm{in})$. \\
$\mathrm{H}$ & 3.50 & 1.73 \\
$\mathrm{dV}$ & 7.55 & 3.65 \\
$\mathrm{dH}$ & 7.55 & 3.65 \\
$\mathrm{dT}$ & 15.10 & 4.05 \\
dmax & 10.33 & 2.70 \\
VarV & 7.53 & 3.55 \\
VarH & 7.53 & 3.55 \\
Latency & 1.56 & $.26(\mathrm{sec})$ \\
\hline
\end{tabular}

and a $t$ test did not reject the equality of the two overall means.

An evaluation of the seven measures as predictors of latency was accomplished through the vehicle of correlation, each measure being correlated with the dependent variable of latency. Both $\mathrm{V}$ and $\mathrm{H}$, while correlating .03 with each other, had a very low and insignificant correlation with latency $(-.18)$, leading to the supposition that vertical or horizontal extent, both of which were based solely on the pattern characteristics, were useless as predictors of latency in a discrimination task. Since it might quite validly be argued that the range of scores in each measure and their standard deviation could produce spurious correlations, the following data should be noted: $\mathrm{V}$ and $\mathrm{H}$ had a range of 6 points and took on six different values; $\mathrm{dV}$ and $\mathrm{dH}$ had a range of 13 points and took on all values in the range; dmax similarly had all values in a 10 -point range, while dT covered a 12-point range; VarV and VarH each had six scores in a 16-point range (three original and three different values from the set inversion). It is therefore possible, since $\mathrm{Var} V$ and $\mathrm{VarH}$ could range over only six values (corresponding to stimulus sets), that correlation would be less appropriate as an analytic technique than a comparison of mean latency under different values of VarV and VarH. When an analysis of variance was performed for groups having different $\mathrm{VarV}$ and VarH values (i.e., the original stimulus sets), the resulting $F$ statistic was less than unity, and the lack of significant differences supports the conclusions arrived at from correlational analysis.

An examination of Table 2, where the intercorrelations of the four measures (based on both the pattern and the domain) as well as the dependent variable are recorded, will show that $\mathrm{dV}$ and $\mathrm{dH}$ do not relate significantly to latency. Ss, therefore, are not consistent in the utilization of variance in either the vertical or the horizontal dimension. The total deviation, however, as well as dmax, which was intended to reflect selective attention, are significantly related to latency while being significantly related to each other.

Since dT and dmax were significantly related, it is not sufficient to show that dmax has the higher correlation with latency in an attempt to prove the importance of selective attention mechanisms. Semipartial correlation coefficients, however, do provide the required supporting evidence. When $\mathrm{dT}$ was partialed out of dmax and the residuals were correlated with latencies, a semipartial correlation of -.32 (significant at the $5 \%$ level) was the result. When, in the opposite case, dmax was partialed out of $\mathrm{dT}$, the resulting correlation was .003 (or, effectually, 0.0). It might then be argued that $\mathrm{dT}$ is predictive of latency only to the extent that it reflects dmax-the measure generated on the assumption of selective attention, filtering, and gating.

It only remains, therefore, to demonstrate the superiority of dmax over VarV and VarH (the domain measures), in order to completely demonstrate the selection effect for the total experiment. VarV correlated -.08 with latency, while VarH had a correlation of -.10 , and in neither case was the relationship significant.

Even though a pilot study indicated the absence of a vertical-horizontal illusion, it was found that the correlation of dmax with latency when $d \max =d V \quad(N=16)$ was -.70 , while the correlation when $\mathrm{dmax}=\mathrm{dH}(\mathrm{N}=14)$ was only -.52 . It is possible that Ss were not as efficient in the utilization of horizontal information as they were in the usage of vertical cues.

Finally, when $\mathrm{dV}$ and $\mathrm{dH}$ were transformed into standardized $\mathrm{Z}$ scores within each pattern set, and dmax was chosen from these distributions, it had a correlation of -.56 with latency. Obviously in cases with dimensions having different standard deviations, $\mathrm{Z}$ scores at some level rather than absolute scores should be the basis of choice. Since the standard deviations for $\mathrm{dH}$ and $\mathrm{dV}$ were identical over all 30 patterns, as was the mean, $Z$ scores applied to the total 30 stimuli would yield no difference in the choice and/or relative magnitude of dmax.

\section{DISCUSSION}

Results of the experiment have supported the contention that Ss engage in selective shifts of attention, even in a series of rapidly presented and highly similar problems where an immediate response was demanded, since dmax was superior to all

Table 2

Intercorrelations of Four Measures Relating the Pattern to the Domain and Latency

\begin{tabular}{lccccc}
\hline Variables & $\mathrm{dV}$ & $\mathrm{dH}$ & $\mathrm{dmax}$ & $\mathrm{dT}$ & Latency \\
\hline $\mathrm{dV}$ & 1.00 & $-.57^{* *}$ & $.42^{* *}$ & $.46^{* *}$ & -.18 \\
$\mathrm{dH}$ & & 1.00 & $.39^{* *}$ & $.46^{* *}$ & -.29 \\
$\mathrm{dmax}$ & & & $.87^{* *}$ & $-.61^{* *}$ \\
$\mathrm{dT}$ & & & & $-.51^{* *}$ \\
$\quad$ Latency & & & & & 1.00 \\
\hline
\end{tabular}


other measures of the pattern and domain in predicting latency. In experiments on multidimensional visual stimulation utilizing different patterns and different responses, it is possible that the selection effect would not be so pronounced, and Ss would be more consistent in their usage of available cues. These results could be interpreted as supporting the multidimensional character of patterns-with the modification that $\mathrm{Ss}^{\text {' }}$ responses to a multidimensional pattern might be unidimensional in cases where selective attention is present.

It should be noted, finally, that dmax is highly predictive of latency not only because it was hypothesized that attention would shift, but because it was assumed that the measure relative to a discrimination task would relate the pattern to the domain rather than reflect the attributes of the pattern or the domain independently. In fact, when the maximum measure was chosen on the basis of $V$ and $H$ (rather than $\mathrm{dV}$ and $\mathrm{dH}$ ), its relationship with latency was effectually zero $(-.04)$, and the same level of correlation was obtained $(-.07)$ when the maximum measure was based on VarV and $\mathrm{VarH}$.

\section{REFERENCES}

AIKEN, L. S., \& BROWN, D. R. A spatial analysis of the discriminability of forms in noise. Perception \& Psychophysics, 1969, 5, 171-175.

BEACH, L. R. Cue probabilism and inference behavior. Psychological Monographs, 1964a, 78(Whole No. 582).

BEACH, L. R. Recognition, assimilation, and identification of objects. Psychological Monographs, 1964b, 78(Whole No. 583).
BEHRMAN, B. W., \& BROWN, D. R. Multidimensional scaling of form: A psychophysical analysis. Perception \& Psychophysics, 1968, 4, 19-25.

BROWN, D. R., \& ANDREWS, M. H. Visual form discrimination: Multidimensional analysis. Perception \& Psychophysics, 1968, 3, 401-406.

BROWN, D. R., \& OWEN, D. H. The metrics of visual form: Methodological dyspepsia. Psychological Bulletin, 1967, 68, 243-259.

DEMBER, W. N. The psychology of perception. New York: Holt, Rinehart, \& Winston, 1960

EDMONDS, E. M., \& EVANS, S. H. Schema learning without a prototype. Psychonomic Science, 1967, 8, 239-240.

EGETH, H. Selective attention. Psychological Bulletin, 1967, 67, 41-57.

EVANS, S. H. Redundancy as a variable in pattern perception. Psychological Bulletin,

FITTS, P. M., \& BIEDERMAN, I. S-R compatability and information reduction. Journal of Experimental Psychology, 1965, 69, 408-412.

IMAI, S., \& GARNER, W. R. Discriminability and preference for attributes in free and constrained classification. Joumal of Experimental Psychology, 1965, 69, 596-608.

KRUSKAL, J. B. Multidimensional scaling for optimizing goodness of fit to a non-metric hypothesis. Psychometrika, 1964a, 29, 1-27.

KRUSKAL, J. B. Non-metric multidimensional scaling: A numerical method. Psychometrika 1964b, 29, 115-129.

MAVRIDES, C. M., \& BROWN, D. R. Discrimination and reproduction of patterns Feature measures and constraint redundancy as predictors. Perception \& Psychophysics, 1969, 6, 276-280.

MORIN, R. E., FORRIN, B., \& ARCHER, W. Information processing behavior: The role of irrelevant stimulus information. Journal of Experimental Psychology, 1961, 61, 89-96.

STENSON, H. H. The psychophysical dimensions of similarity among random shapes. Perception \& Psychophysics, 1968, 3, 201-214.

TORGERSON, W. S. Theory and methods of scaling. New York: Wiley, 1958. $1967,67,104-113$.

subsequently recognized in a normal, upright orientation than (1) when faces are viewed and recognized in an inverted orientation or (2) when faces are initially viewed in an inverted, and subsequently recognized in an upright, orientation. These results were interpreted as suggesting that faces are not coded and stored simply on the basis of their pattern characteristics. Yin (1969) reported similar findings and drew similar conclusions.

It is possible, however, that the specific transformation employed in those investigations, i.e., inversion, introduces some artifacts peculiar to the results of inversion. For example, perhaps the salient properties by which faces as patterns differ from each other are specific to their upper regions, and perhaps subjects attend primarily to the upper regions of any pattern when viewing it with the intention of remembering it (see, e.g., Ghent, 1960; Braine, 1965). This combination of factors would then, when pictures of faces are inverted, result in a decrement of recognition accuracy in the same way that it would for any set of patterns which were to be distinguished among on the basis of cues that appear in an "unfavored" position.

The present study addresses itself to circumventing this possibility by applying a transformation in which brightness relationships are reversed. Photographic negatives are employed, thus maintaining both pattern and orientation, but changing the visual characteristics into a form in which faces are not normally viewed. This particular transformation is also suggested by Köhler's (1940) speculation that the impaired recognizability of inverted pictures of faces might be attributable to the difficulty, under those circumstances, of determining their expressions. As there is, similarly, informal evidence that the determination of expression is greatly impaired when faces are seen in photographic negative (e.g., Hebb, 1949; Hochberg, 1968), investigation of face recognition under these conditions would seem to be a logical and appropriate step. Impairment of recognition accuracy for faces seen in negative would thus both provide more substantial support for the hypothesis that pictures of faces are not recognized simply as patterns, but rather, also on the basis of some factors specific to faces as such, and suggest that one of these factors be the susceptibility of a face to be characterized by an expression.

\section{SUBJECTS}

Twenty-four undergraduate students (12

Hochberg \& Galper (1967) have male, 12 female) at New York University demonstrated that recognition accuracy for served as Ss.

faces seen in still photographs is higher when faces are initially viewed and then

\section{MATERIALS}

Fifty photographs of females were 